\title{
Alternative models of disorders of traumatic stress based on the new ICD-11 proposals
}

\author{
Shevlin M, Hyland P, Karatzias T, Fyvie C, Roberts N, Bisson JI, \\ Brewin CR, Cloitre M. Alternative models of disorders of traumatic \\ stress based on the new ICD-11 proposals.
}

Objective: Although there is emerging evidence for the factorial validity of the distinction between post-traumatic stress disorder (PTSD) and complex PTSD (CPTSD) proposed in ICD-11, such evidence has been predominantly based on using selected items from individual scales that describe these factors. We have attempted to address this gap in the literature by testing a range of alternative models of disorders of traumatic stress using a broader range of symptoms and standardized measures.

Method: Participants in this cross-sectional study were a sample of individuals who were referred for psychological therapy to a National Health Service (NHS) trauma centre in Scotland $(N=195)$.

Participants were recruited over a period of 18 months and completed measures of stressful life events, DSM-5 PTSD, emotion dysregulation, self-esteem and interpersonal difficulties.

Results: Overall, results indicate that a structural model incorporating six first-order factors (re-experiencing, avoidance of traumatic reminders, sense of threat, affective dysregulation, negative self-concept and disturbances in relationships) and two second-order factors (PTSD and disturbances in self-organization [DSO]) was the best fitting. The model presented with good concurrent validity. Childhood trauma was found to be more strongly associated with DSO than with PTSD.

Conclusion: Our results are in support of the ICD-11 proposals for PTSD and CPTSD.

\author{
M. Shevlin ${ }^{1}$,P. Hyland ${ }^{2}$, \\ T. Karatzias ${ }^{3,4}$, C. Fyvie ${ }^{\prime}$, \\ N. Roberts ${ }^{5}$, J. I. Bisson ${ }^{6}$, \\ C. R. Brewin', M. Cloitre ${ }^{8,9}$
}

${ }^{1}$ School of Psychology, Ulster University, Derry, UK,

${ }^{2}$ School of Business, National College of Ireland, Dublin, Ireland, ${ }^{3}$ School of Health \& Social Care, Edinburgh

Napier University, Edinburgh, ${ }^{4}$ Rivers Centre for

Traumatic Stress, NHS Lothian, Edinburgh, ${ }^{5}$ Psychology

and Counselling Directorate, Cardiff and Vale University

Health Board, Cardiff, ${ }^{6}$ School of Medicine, Cardiff

University, Cardiff, ${ }^{7}$ Clinical, Education \& Health

Psychology, University College London, London, UK,

${ }^{8}$ School of Medicine, New York University, New York,

NY, and ${ }^{9}$ National Center for PTSD, Veterans Affairs

Palo Alto Health Care System, Palo Alto, CA, USA

Key words: post-traumatic stress disorder; complex posttraumatic stress disorder; disorders of traumatic stress; ICD-11

Thanos Karatzias, Edinburgh Napier University, Sighthill Campus, Sighthill Court, Edinburgh EH11 4BN, UK.

E-mail: t.karatzias@napier.ac.uk

Accepted for publication December 20, 2016

\section{Significant outcomes}

- A structural model incorporating six first-order factors and two second-order factors (PTSD and DSO) provided the best fit to the data.

- Results are in support of the ICD-11 proposals for CPTSD.

\section{Limitations}

- Sample consisted predominantly of people who had experienced childhood psychological trauma or been multiply traumatized in childhood and adulthood.

- A number of possible alternative symptoms in the tested models that are highly prevalent in trauma populations, such as borderline symptoms and dissociation, were not considered.

\section{Introduction}

Two 'sibling disorders' have been proposed for ICD-11: post-traumatic stress disorder (PTSD) and complex PTSD (CPTSD) (1). The organizing principles for the ICD-11 revisions were that diagnoses should be consistent with clinicians' mental health taxonomies, limited in the number of symptoms included and based on distinctions important for management and treatment (2). The ICD-11 model of PTSD includes symptoms reflecting three clusters: (i) re-experiencing of the 


\section{Shevlin et al.}

trauma in the present, (ii) avoidance of traumatic reminders and (iii) a persistent sense of threat that is manifested by increased arousal and hypervigilance. These symptoms define PTSD as a response characterized by some degree of fear or horror related to a specific traumatic event. In contrast, the symptom profile of CPTSD includes the core PTSD symptoms plus additional symptoms that identify 'disturbances in self-organization' (DSO) which may result from sustained, repeated and multiple forms of traumatic exposures. There are three DSO symptom categories: (i) affective dysregulation, (ii) negative self-concept and (iii) disturbances in relationships. Thus, the ICD-11 proposals contain two logically distinct elements: a structural description of PTSD and CPTSD involving two groups of three factors, and new content concerning the key symptoms. This study addresses the structural element of the proposals.

There have been numerous factor analytic studies that have provided evidence for this conceptualization of PTSD and CPTSD. Support for the three-factor structure of PTSD is a necessary prerequisite for the validity of the CPTSD model. Confirmatory factor analytic studies using different measures (e.g. Clinician-Administered PTSD Scale, Harvard Trauma Questionnaire, PTSD Checklist for DSM-5) with various clinical and community samples (e.g. survivors of childhood trauma, refugees, traumatic injury survivors admitted to hospital, bereaved parents) have provided evidence in support of this structure (3-6). Furthermore, there is evidence that indicators of DSO are also best explained in terms of the three correlated factors described in ICD-11, based on data from adult survivors of childhood institutional abuse (7) and treatment-seeking victims of interpersonal violence ranging from prolonged childhood abuse to adult single-incident traumas (8).

The ICD-11 proposes that CPTSD is multidimensional and hierarchical, that is that the three symptom clusters defining PTSD can be explained by a higher order 'PTSD' factor and that affective dysregulation, negative self-concept and disturbances in relationships can be explained by a higher order 'DSO' factor. It would also be expected that these superordinate PTSD and DSO factors would be positively correlated. Hyland et al. (9) tested this hypothesis using data from a large sample of treatmentseeking adult victims of childhood sexual abuse and found that, of four alternative models tested, the ICD-11 model was the best fitting, and the PTSD and DSO factors were strongly correlated $(r=0.81)$. Tay et al. (10) also reported acceptable fit for a multidimensional solution with the six correlated first-order factors based on data from West Papuan refugees. However, a model with two second-order factors was not tested in this research.

To date, the extant research evidence appears to support the construct validity of the ICD-11 model of CPTSD. However, a salient limitation of all previous studies has been the ad hoc approach to the measurement of the three DSO dimensions that comprise the CPTSD diagnosis. The absence of a specific measure of ICD-11 CPTSD symptomatology has meant that previous studies have had to rely on the selection of items from pre-existing scales such as the Brief Symptom Inventory [BSI (11)] and the Trauma Symptom Checklist [TSC (12)] to capture the proposed DSO symptoms. Although this approach offered a viable method of assessing the structure of CPTSD, such an approach fails to recognize and capture the dimensional nature of the three DSO constructs of affective dysregulation, negative selfconcept and disturbances in relationships. An alternative approach to modelling the DSO constructs, and by extension testing the structural validity of the proposed CPTSD construct, is through the use of dimensional measures that can more accurately capture the continuous distribution of scores on each DSO dimension. Specifically, in this study PTSD symptoms were measured using the PTSD Checklist for DSM-5 [PCL-5 (13)], while affective dysregulation was assessed using the Difficulties in Emotion Regulation Scale [DERS (14)], negative self-concept by (low scores on) the Rosenberg Self-Esteem Scale [RSES (15)] and disturbances in relationships using the Inventory of Interpersonal Problems [IIP (16)].

Effectively, therefore, this study tested the structural proposals for CPTSD contained in ICD-11 independently of the specific symptomatology content proposals. It was predicted that the model with six first-order factors and two second-order factors (PTSD and DSO) would be well-fitting and would fit better than alternative models that were not both multidimensional and hierarchical. Furthermore, the concurrent validity of the ICD-11 model was tested by estimating the associations between the first- and second-order PTSD and DSO factors with scores reflecting exposure to childhood trauma. To be consistent with the ICD-11 proposals, it was predicted that childhood trauma would be more strongly associated with DSO than with PTSD. 


\section{Methods}

Participants and procedure

Participants in this study were individuals who were referred by general practitioners, psychiatrists or psychologists for psychological therapy to a National Health Service (NHS) trauma centre in Scotland. All 230 new patients over the 18-month recruitment period were sent a letter and invited to complete a set of standardized measures. Twentytwo did not respond and 13 provided unusable data due to large amounts of missing responses, which resulted in a final sample size of 195 . Ethics approval for the study was sought by the NHS Lothian Committee.

The mean age of the sample was 41 years $(\mathrm{SD}=12.4)$, and there were more females $(65.1 \%)$ than males. Most of the sample were born in the United Kingdom (88.7\%), and of these, most were from Scotland (79\%). The highest level of academic attainment was varied: school (38.5\%), college (30.2\%) and university (30.2\%). Approximately one-third of the sample were in employment (full-time 20.2\%, part-time 13\%), 38.9\% were unemployed, $7.3 \%$ were retired and 5.7\% were in voluntary work (15\% reported 'None of these'). Almost half of the sample were single $(48.2 \%), 22.3 \%$ were married, $12.4 \%$ were divorced, 9.8\% were cohabiting and 7.3\% indicated 'Other'. Most participants were either living with a partner or with their family (41\%) and $34.7 \%$ were living alone (and 24.4\% reported 'Other').

\section{Measures}

PTSD checklist for DSM-5 (PCL-5). The PCL-5 is a 20-item self-report measure that assesses the 20 DSM-5 symptoms of PTSD (13). Initially the PCL-5 assesses Criterion A with the following instructions, 'This questionnaire asks about problems you may have had after a very stressful experience involving actual or threatened death, serious injury, or sexual violence. It could be something that happened to you directly, something you witnessed, or something you learned happened to a close family member or close friend. Some examples are a serious accident; fire; disaster such as a hurricane, tornado, or earthquake; physical or sexual attack or abuse; war; homicide; or suicide. First, please answer a few questions about your worst event, which for this questionnaire means the event that currently bothers you the most'. The scale instructions are, 'Keeping your worst event in mind, please read each problem carefully and then circle one of the numbers to the right to indicate how much you have been bothered by that problem in the past month'. Participants respond using a 5-point Likert scale, ranging from 'Not at all' (0) to 'Extremely' (4). Symptom cluster severity scores are calculated for Intrusions (five items), Avoidance (two items), Negative alterations in cognitions and mood (seven items) and Alterations in arousal and reactivity (six items). For the purposes of the current study, only selected items from the Intrusions (B2, B3 and B4), Avoidance (C1 and C2) and Arousal (E3 and E4) clusters were selected to capture the ICD-11 PTSD symptom profile. The scale can be used to generate a self-report DSM-5 diagnosis using a cut-point of 38. Studies have reported acceptable psychometric properties of the PCL-5 scores in non-clinical (17) and trauma-exposed samples (18). Wilkins et al. (19) provide a comprehensive review of the psychometric evaluation of earlier versions of the PCL. Reliability estimates (composite reliability) of the Intrusion (0.70) and Arousal (0.78) item scales were moderate but lower for Avoidance (0.50).

Difficulties in emotion regulation scale. The DERS is a standardized 36-item measure of emotion regulation involving not just the modulation of emotional arousal, but also the awareness, understanding and acceptance of emotions, and the ability to act in desired ways regardless of emotional state (14). It provides six subscales including 'Non-acceptance of emotional responses', 'Difficulties in engaging in goal directed behaviour', 'Impulse control difficulties', 'Lack of emotional awareness', 'Limited access to emotional regulation strategies' and 'Lack of emotional clarity'. Participants are asked to indicate how often the items apply to themselves, with responses ranging from 'Almost never' (1) to 'Almost always' (5). In this study, the mean scores on the 'Impulse control difficulties' and 'Lack of emotional awareness' scales were used. The DERS subscales have been found to be unidimensional (20), and the reliability of the scales was high in this sample: 'Impulse control difficulties' (0.89) and 'Lack of emotional awareness' $(0.83)$.

Rosenberg self-esteem scale. The RSES consists of 10 Likert-type scale items designated to assess positive and negative evaluations of self (15). Respondents indicate their level of agreement ranging from 1 (strongly disagree) to 4 (strongly agree). Thus, the possible total score can range from a minimum of 10 to a maximum of 40 , with higher scores reflecting more positive evaluations of self. Based on data from 16998 participants across 53 nations, a score of $<26$ was considered to represent 


\section{Shevlin et al.}

low self-esteem (21). The RSES has been found to be unidimensional (22), and the reliability of the scale scores was high in this sample (0.89).

Inventory of interpersonal problems - short circumplex form (IIP). The Inventory of Interpersonal Problems - Short Circumplex Form (IIP) is a 32-item self-report measure of interpersonal difficulties and consists of eight subscales (Domineering, Vindictive, Cold, Socially Avoidant, Non-assertive, Exploitable, Overly Nurturant and Intrusive) with responses based on a 5-point Likert scale ranging from 'Almost never' (1) to 'Almost always' (5). In this study, the 'Socially Avoidant' and 'Cold' scales were used (16). In a large sample of university students, the mean scores for the Socially Avoidant' and 'Cold' subscales were 1.18 and 1.09 respectively (23). The subscales have been found to be unidimensional (24), and the reliability of the scales was high: 'Socially Avoidant' (0.85) and 'Cold' (0.87).

Childhood trauma questionnaire. The Childhood trauma questionnaire (CTQ) is a 28-item selfreport questionnaire that assesses exposure to range of different childhood traumas (25). The scale produces five subscales, each with five items: Emotional Abuse, Physical Abuse, Sexual Abuse, Emotional Neglect and Physical Neglect. Items are responded to using a 5-point scale ranging from 'never true' (1) to 'very often true' (5) which produces possible scores 5-25 for each trauma subscale. The reliability of the scales was high in this sample: Emotional Abuse (0.90), Physical Abuse (0.85), Sexual Abuse (0.97), Emotional Neglect (0.92) and Physical Neglect (0.83).

The life events checklist. The life events checklist (LEC) is a 17-item self-report measure designed to screen for potentially traumatic events in a respondent's lifetime (26). The LEC assesses lifetime exposure to 16 traumatic events (e.g. Natural disaster, Physical assault, Life threatening illness/injury), and the 17th item, 'Any other very stressful event/experience', can be used to indicate exposure to a trauma that was not listed. For each item, the respondent checks whether the event 'Happened to me' (1), 'Witnessed it happening to somebody else' (2), 'Learned about it happening to someone close to me' (3), 'Part of my job' (4), 'Not sure it applies' (5) and 'Doesn't apply to my experience' (6). In order to create a summed total to represent the number of different life events that have been experienced, the items were recoded into binary variables with 'Happened to me' responses being coded as 1 and all other responses coded as 0 . This produced a single 'Total traumas' variable with possible scores ranging from 0 to 16 ; item 17 was not included as the nature of the trauma could not be identified.

Analysis

The latent structure of CPTSD was tested using confirmatory factor analysis (CFA). PTSD symptoms were measured using seven items from the PCL-5 that measure each of the ICD-11 PTSD symptoms clusters. Item-level analysis for the DSO variables (Emotion Regulation Scale, the Rosenberg Self-Esteem Scale and the Inventory of Interpersonal Problems) was not appropriate given the large number of items and the modest sample size. To ensure that each DSO latent variable had multiple indicators, thereby controlling for measurement error, the models were specified using item parcels. Item parcelling is a method by which individual items for a construct are combined into a small number of 'parcels' that can then be used as observed variables in the CFA. Items can be parcelled in different ways; for example, an 8-item scale (Q1-Q8) could be parcelled into two observed variables by taking the sum or average of the first four and last four items (e.g. Parcel $1=$ Sum Q1 to Q4, Parcel $2=$ Sum Q5 to Q8), using odd/even numbered items (e.g. Parcel $1=$ Sum Q1, Q3, Q5, Q7; Parcel 2 = Sum Q2, Q4, Q6, Q8), or random allocation (e.g. Parcel $1=$ Sum Q1, Q2, Q5, Q8; Parcel 2 = Sum Q3, Q4, Q6, Q7). Matsunaga (27) provides a comprehensive overview on the benefits, and potential limitations, of using item parcels in latent variable modelling. Table 1 provides details on the PCL items that were used and how the items for all the DSO measures were parcelled.

The choice of DERS and IIP subscales was made to be consistent with the indicators previously used to measure the affective dysregulation and disturbances in relationships factors (see 28, 29). In addition, the affective dysregulation dimension has been considered to represent both emotional hyperactivation and deactivation, and the DERS 'Impulse control difficulties' and 'Lack of emotional awareness' subscales reflect these dimensions respectively. Ford et al. (30) noted that two important aspects of the disturbances in relationships construct are difficulties in sustaining relationships and difficulty in maintaining emotional engagement; the 'Socially Avoidant' and 'Cold' subscales from the IIP were used to reflect these aspects.

Seven alternative models were specified (see Fig. 1) and tested. Model 1 is a one-factor model 
Models of disorders of traumatic stress

Table 1. Description of measures, items and item parcels used in CFA of complex PTSD

\begin{tabular}{|c|c|c|c|}
\hline Symptom cluster & Measure & Items/Parcels & Items/Example items \\
\hline \multirow{3}{*}{ Re-experiencing } & \multirow[t]{3}{*}{ PCL-5 Intrusion scale } & Re1 = PCL Intrusion item 2 & Repeated, disturbing dreams of the stressful experience? \\
\hline & & Re2 $=$ PCL Intrusion item 3 & $\begin{array}{l}\text { Suddenly feeling or acting as if the stressful experience were actually } \\
\text { happening again (as if you were actually back there reliving it)? }\end{array}$ \\
\hline & & Re3 = PCL Intrusion item 4 & Feeling very upset when something reminded you of the stressful experience? \\
\hline \multirow[t]{2}{*}{ Avoidance } & \multirow[t]{2}{*}{ PCL-5 Avoidance scale } & Av1 = PCL Avoidance item 6 & Avoiding memories, thoughts, or feelings related to the stressful experience? \\
\hline & & Av2 = PCL Avoidance item 7 & $\begin{array}{l}\text { Avoiding external reminders of the stressful experience (for example, people, } \\
\text { places, conversations, activities, objects, or situations)? }\end{array}$ \\
\hline \multirow[t]{2}{*}{ Sense of Threat } & \multirow[t]{2}{*}{ PCL-5 Arousal scale } & Th1 = PCL Arousal item 17 & Being 'superalert' or watchful or on guard? \\
\hline & & Th2 $=$ PCL Arousal item 18 & Feeling jumpy or easily startled? \\
\hline \multirow[t]{2}{*}{ Affective dysregulation } & \multirow[t]{2}{*}{$\begin{array}{l}\text { Difficulties in emotion } \\
\text { regulation scale }\end{array}$} & $\begin{array}{l}\text { AD1 = mean of DERS 'Impulse' } \\
\text { subscale }\end{array}$ & $\begin{array}{l}\text { When I'm upset, I lose control over my behaviours. } \\
\text { I experience my emotions as overwhelming and out of control. }\end{array}$ \\
\hline & & $\begin{array}{l}\mathrm{AD} 2=\text { mean of DERS 'Clarity' } \\
\text { subscale }\end{array}$ & $\begin{array}{l}\text { I have difficulty making sense out of my feelings. } \\
\text { I have no idea how I am feeling. }\end{array}$ \\
\hline \multirow[t]{2}{*}{ Negative Self-Concept } & \multirow[t]{2}{*}{$\begin{array}{l}\text { Rosenberg self-esteem } \\
\text { scale }\end{array}$} & $\begin{array}{l}\text { NSC1 }=\text { sum of Rosenberg items } \\
1,3,5,7,9\end{array}$ & On the whole, I am satisfied with myself. \\
\hline & & $\begin{array}{l}\text { NSC2 }=\text { sum of Rosenberg items } \\
2,4,6,8,10\end{array}$ & At times I think I am no good at all. \\
\hline \multirow[t]{2}{*}{ Disturbances in relationships } & \multirow[t]{2}{*}{$\begin{array}{l}\text { Inventory of interpersonal } \\
\text { problems }\end{array}$} & $\begin{array}{l}\text { DR1 }=\text { mean of IIP 'Socially } \\
\text { avoidant' subscale }\end{array}$ & $\begin{array}{l}\text { It is hard for me to introduce myself to new people. } \\
\text { It is hard for me to socialise with other people. }\end{array}$ \\
\hline & & $\begin{array}{l}\text { DR1 = mean of IIP 'Cold' } \\
\text { subscale }\end{array}$ & $\begin{array}{l}\text { It is hard for me to feel close to other people. } \\
\text { I keep people at a distance too much. }\end{array}$ \\
\hline
\end{tabular}

where all PTSD and DSO items load on a single latent variable. Model 2 is a correlated six-factor model (Intrusion, Avoidance, Arousal, Affective dysregulation, Negative self-concept and Disturbances in relationships). Model 3 replaced the factor correlations in Model 2 with a single secondorder factor representing CPTSD. Model 4 specified two correlated second-order factors (PTSD and DSO) to explain the covariation among the six first-order factors: Re-experiencing, Avoidance and Threat loaded on the PTSD factor, and Affective dysregulation, Negative self-concept and Disturbances in relationships loaded on the DSO factor. Models 5 and 6 tested the hypotheses that there was (1) no hierarchical structure for the PTSD items but a hierarchical structure for the DSO items (Model 5), and (2) no hierarchical structure for the DSO items but a hierarchical structure for the PTSD items (Model 6). Model 7 proposed that all the PTSD and DSO items loaded on two correlated first-order factors. For all models, the error variances were uncorrelated.

Each model was specified and estimated in Mplus 7.1 (31) using robust maximum-likelihood estimation [MLR (32)] which has been shown to produce correct parameter estimates, standard errors and test statistics (33). The MLR estimator is appropriate when the data are interval, such as the DSO item parcel variables, or ordinal with more than three categories (34). The proportion of missing data for all study variables ranged from 0 to $3.6 \%$, with a mean of $1.84 \%$. For the confirmatory factor analysis, this was handled using all available information which is the default when the MLR estimator is used (31). Goodness of fit for each model was assessed with a range of fit indices including the chi-square $\left(\chi^{2}\right)$, the comparative fit index [CFI (35)] and the Tucker-Lewis index [TLI (36)]. A non-significant $\chi^{2}$ and values $>0.90$ for the CFI and TLI were considered to reflect acceptable model fit. Additionally, the root mean square error of approximation [RMSEA (37)] was reported, where a value $<0.05$ indicated close fit and values up to 0.08 indicated reasonable errors of approximation (38). The same cut-off values can be used for the standardized root mean square residual [SRMR (38)]. The Bayesian information criterion [BIC (39)] was also used to assess the relative fit of the models. The model with the lowest BIC was considered to be the better model, and a difference $>10$ was considered to be indicative of a 'significant' difference (40). When the best model was identified, factor scores were calculated (41), and these were correlated with the criterion variables.

\section{Results}

\section{Sample characteristics}

The prevalence of DSM-5 PTSD, based on a cutoff score over 38 on the PCL-5, was high at $88.2 \%$ and did not differ by gender (male $=92.6 \%$, female $\left.=85.8 \%, \chi^{2}(1)=1.98, P>0.05\right)$. Medication had been prescribed to $67.5 \%$ of the sample. The participants also reported exposure to multiple traumatic events. The median number of traumas reported using the Life Events Checklist was 5 


\section{Shevlin et al.}
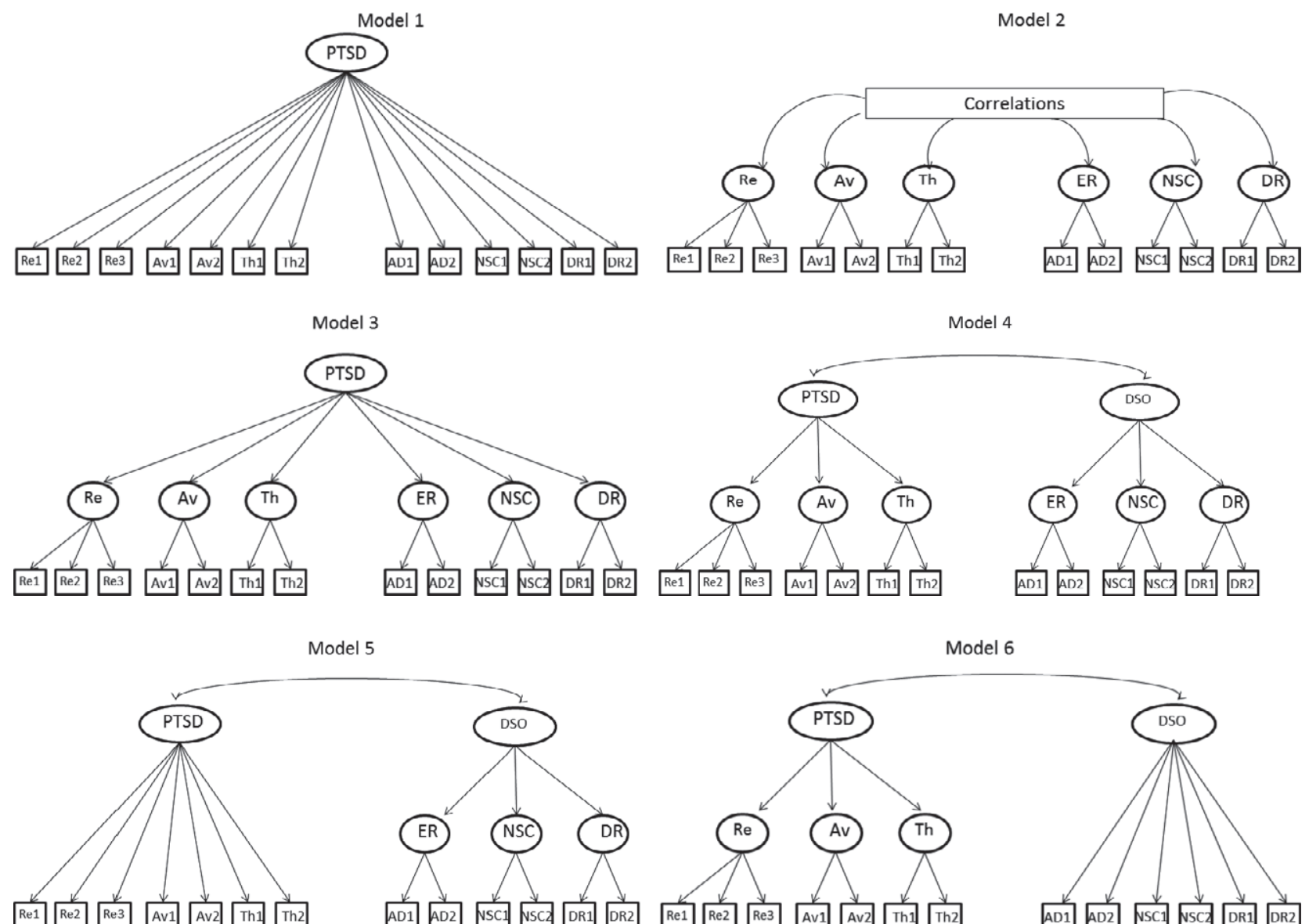

Model 5
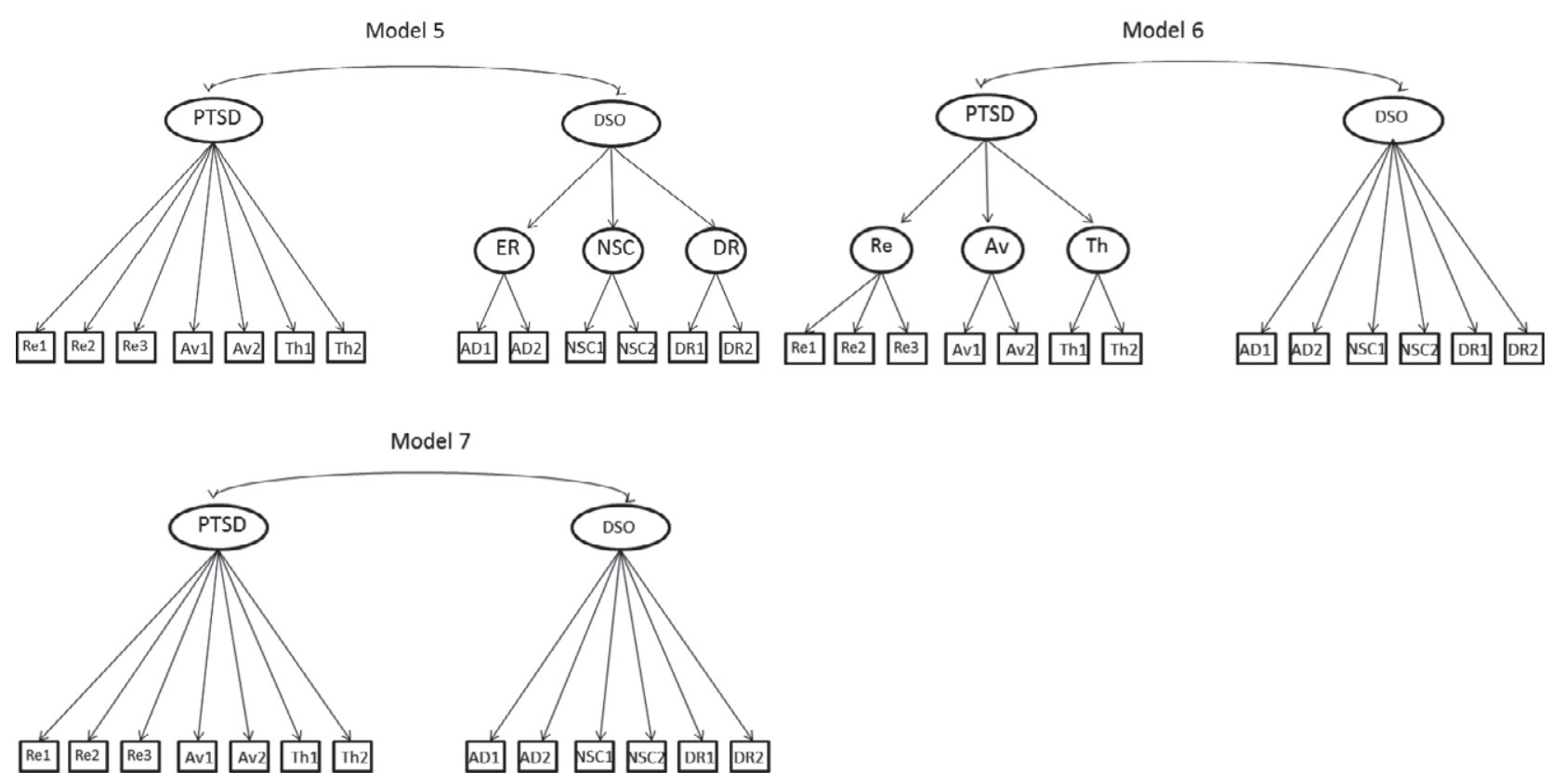

Fig. 1. Alternative factor models of the PTSD and DSO variables. Re1, Re 2, Re 3 = PCL Re-experiencing items 2, 3, 4; Av1, Av2 = PCL Avoidance items 6, 7; Th1, Th2 = PCL Threat items 17, 18; AD1 = mean of DERS 'Impulse' subscale; AD2 = mean of DERS 'Clarity' subscale; NSC1 = sum of Rosenberg items 1, 3, 5, 7, 9; NSC2 $=$ sum of Rosenberg items 2, 4, 6, 8, 10; DR1 = mean of IIP 'Socially avoidant' subscale; DR2 = mean of IIP 'Cold' subscale.

(25th percentile $=3, \quad 75$ th $\quad$ percentile $=7$, $\mathrm{IQR}=4)$, and there were no significant differences between males and females (median test $(1)=0.07$, $P>0.05)$. Only a small number $(4.6 \%)$ reported exposure to a single traumatic event; $71.8 \%$ of the sample reported experiencing between three and eight traumatic events. Descriptive statistics for the main study variables are presented in Table 2 .

The mean scores on the PTSD items were all above the scale mid-point of 2 ('Moderately' bothered by symptom), and many closer to, or above, 3
('Quite a bit' bothered by symptom), and there were no significant gender differences. The mean scores for the 'Impulse' and 'Clarity' subscales of the DERS were approximately 3 , and this corresponds to the rating emotional difficulties 'About half the time'. Males scored significantly higher on the 'Clarity' subscale, and the effect size was small to moderate (Cohen's $d=0.34$ ). The mean scores for the Rosenberg Self-esteem Scale were lower than the cut-off score that indicates 'low' selfesteem, and there were no significant gender 
Models of disorders of traumatic stress

Table 2. Descriptive statistics for main study variables

\begin{tabular}{lrrr}
\hline Measure & \multicolumn{1}{c}{ Male } & \multicolumn{1}{c}{ Female } & \multicolumn{1}{c}{ Total } \\
\hline PTSD & & & \\
Re1 (PCL item 2) & $2.50(1.33)$ & $2.63(1.36)$ & $2.58(1.35)$ \\
Re2 (PCL item 3) & $2.27(1.40)$ & $2.48(1.43)$ & $2.41(1.42)$ \\
Re3 (PCL item 4) & $3.04(0.99)$ & $3.22(1.02)$ & $3.15(1.01)$ \\
Av1 (PCL item 6) & $3.11(1.07)$ & $3.20(1.01)$ & $3.17(1.03)$ \\
Av2 (PCL item 7) & $2.97(1.09)$ & $2.97(1.19)$ & $2.97(1.15)$ \\
Th1 (PCL item 17) & $3.14(1.04)$ & $2.93(1.37)$ & $3.01(1.27)$ \\
Th2 (PCL item 18) & $2.85(1.24)$ & $2.86(1.29)$ & $2.86(1.27)$ \\
DSO & & & \\
DERS 'Impulse' subscale & $2.92(0.99)$ & $2.76(1.11)$ & $2.81(1.07)$ \\
DERS 'Clarity' subscale & $3.13^{*}(0.76)$ & $2.84^{*}(0.91)$ & $2.94(0.88)$ \\
Rosenberg Self-esteem Scale & $20.73(5.02)$ & $21.33(6.22)$ & $21.12(5.81)$ \\
IIP 'Socially avoidant' subscale & $2.73(1.00)$ & $2.66(1.11)$ & $2.69(1.07)$ \\
IIP 'Cold' subscale & $2.38^{*}(1.04)$ & $1.88^{*}(1.23)$ & $2.06(1.19)$ \\
Childhood Trauma & & & \\
CTQ: Emotional abuse & $12.66^{*}(6.40)$ & $15.06^{*}(6.70)$ & $14.19(6.67)$ \\
CTQ: Physical abuse & $10.95(5.91)$ & $10.50(5.90)$ & $10.67(5.89)$ \\
CT0: Sexual abuse & $10.08^{*}(7.22)$ & $13.78^{*}(8.25)$ & $12.44(8.07)$ \\
CTQ: Emotional neglect & $12.77(6.34)$ & $13.89(6.17)$ & $13.48(6.24)$ \\
CT0: Physical neglect & $8.92(4.93)$ & $9.88(5.04)$ & $9.53(5.01)$ \\
\hline
\end{tabular}

${ }^{*}$ Mean difference between males and females significant using $t$-test $(P<0.05)$. PCL, PTSD Checklist for DSM-5; DERS, Difficulties in Emotion Regulation Scale; IIP, Inventory of Interpersonal Problems; CTQ, Childhood Trauma Questionnaire.

differences. The mean scores on the 'Socially avoidant' and 'Cold' subscales were higher than the non-clinical norms, and males scored significantly higher and the effect size was moderate (Cohen's $d=0.44)$. The mean scores on the CTQ, using the classification developed by the scale's authors (25), represented 'moderate to severe' levels of emotional abuse (females significantly higher but in same category, effect size small to moderate, Cohen's $d=0.34$ ) and physical abuse, and 'Slight to moderate' levels of emotional neglect and physical neglect. Females scored significantly higher than males on the sexual abuse scale, and the effect size was moderate (Cohen's $d=0.48$ ); females scored in the 'Severe to extreme' category and males in the 'moderate to severe' category. Endorsement rates for any item (score $>1$ ) from the CTQ subscales indicated that any experience of childhood trauma was also high: Emotional Abuse 84.6\%, Physical Abuse 63.8\%, Sexual Abuse $53.3 \%$, Emotional Neglect $79.8 \%$ and Physical Neglect $68.6 \%$.

\section{Confirmatory factor analysis}

The fit statistics for the seven models of PTSD and CPTSD are presented in Table 3.

Although the chi-square statistics were statistically significant, this should not lead to the rejection of the models as the power of the chi-square test is positively related to sample size (42). Models 2, 3 and 4 met the criteria for acceptable model fit
Table 3. Fit statistics for the alternative models of CPTSD

\begin{tabular}{lcccccc}
\hline Model & BIC & $\begin{array}{c}\text { Chi-square } \\
\text { (df) }\end{array}$ & RMSEA (90\% Cl) & CFI & TLI & SRMR \\
\hline 1 & 8111.841 & $278.422(65)^{*}$ & $0.130(0.114-0.146)$ & 0.724 & 0.669 & 0.086 \\
2 & 7985.026 & $90.142(50)^{*}$ & $0.064(0.042-0.085)$ & 0.948 & 0.919 & 0.043 \\
3 & 7981.351 & $129.12(59)^{*}$ & $0.078(0.060-0.096)$ & 0.909 & 0.880 & 0.063 \\
4 & 7957.544 & $101.524(58)^{*}$ & $0.062(0.041-0.082)$ & 0.944 & 0.924 & 0.046 \\
5 & 7986.494 & $145.498(61)^{*}$ & $0.084(0.067-0.102)$ & 0.891 & 0.860 & 0.055 \\
6 & 7984.353 & $143.132(61)^{*}$ & $0.083(0.065-0.101)$ & 0.894 & 0.864 & 0.053 \\
7 & 8012.836 & $186.324(64)^{*}$ & $0.099(0.083-0.116)$ & 0.842 & 0.807 & 0.061 \\
\hline & & & & &
\end{tabular}

Df, degrees of freedom; BIC, Bayesian information criteria; CFI, comparative fit index; TLI, Tucker-Lewis index; RMSEA, root mean square error of approximation; SRMR, standardized root mean square residual.

based on the CFI, TLI and RMSEA, and additionally, these models also had the lowest values for the SRMR. These were the best fitting models, and the BIC indicated that Model 4 was better than models 2 and 3; the difference between the BIC values for Model 2 and Model 3 was small $(\triangle \mathrm{BIC}=3.676)$, but the differences between the BIC for Model 4 and Model $2(\Delta \mathrm{BIC}=27.482)$ and between Model 4 and Model 3 $(\triangle \mathrm{BIC}=23.807)$ were larger and $>10$ which is considered to be indicative of a 'significant' difference. Therefore, Model 4 should be considered the best model. The factor loadings for Model 4 are presented in Table 4.

The second-order factor loadings for the PTSD factor $($ Re-experiencing $=0.94$, Avoidance $=0.81$, Threat $=0.70$ ) and the DSO factor (Affective Dysregulation $=0.79$, Negative Self-Concept $=$ $-0.89,{ }^{1}$ Disturbances in relationships $=0.90$ ) were

Table 4. Standardized first-order factor loadings for model 4 of CPTSD

\begin{tabular}{lllllll}
\hline Variable & Re & Av & Th & AD & NSC & DR \\
\hline Re1 & 0.685 & & & & \\
Re2 & 0.601 & & & & \\
Re3 & 0.673 & & & & \\
Av1 & & 0.484 & & & \\
Av2 & & 0.605 & & & \\
Th1 & & 0.717 & & \\
Th2 & & 0.883 & & \\
AD1 & & & 0.747 & & \\
AD2 & & & 0.731 & & \\
NSC1 & & & & 0.866 & \\
NSC2 & & & & & 0.848 \\
DR1 & & & & & \\
DR2 & & & & & \\
\hline
\end{tabular}

All loading statistically significant $(P<0.05)$.

Re, Re-experiencing; Av, Avoidance; Th, Sense of Threat; AD, Affective Dysregulation; NSC, Negative Self-Concept; DR, Disturbances in Relationships.

${ }^{1}$ This factor loading is negative as self-esteem reflects positive self-evaluation and the DSO factor is scored in a negative direction. 


\section{Shevlin et al.}

all high and statistically significant $(P<0.05)$. The correlation between the PTSD and DSO factor was $0.72(P<0.05)$. The correlations between the factor scores derived from Model 4 and the criterion variables are presented in Table 5 .

\section{PTSD/CPTSD correlations}

The childhood trauma scores were positively associated with factor scores for all first-order factors and magnitude of the correlations was quite similar for both the PTSD and DSO variables, although the (absolute) correlations were generally higher for the DSO variables. The differences were larger for the emotional and physical neglect variables. For the second-order factor scores, the correlations were higher for DSO than for PTSD, although the differences in the magnitude of the correlations were small.

Overall, results indicate that Model 4 with six first-order factors and two second-order factors (PTSD and DSO) was the best fitting model. The model presented with good concurrent validity. Childhood trauma was also found to be more strongly associated with DSO than with PTSD.

\section{Discussion}

Emerging evidence assessing the factorial validity of ICD-11 proposals for CPTSD has provided support for both a six-factor correlated model (10) and second-order hierarchical model (9). A limiting factor of existing studies is the use of selected items from individual scales that describe these factors. We have attempted to address this gap in the literature by testing a range of alternative models of CPTSD using standardized measures that more effectively capture the dimensional nature of the three DSO factors. Overall, results indicate that a model with six first-order factors and two secondorder factors (PTSD and DSO) was the best fitting model, with good concurrent validity. The fit of this model to the data was considered acceptable based on established criteria for assessment of model fit (43). Our results add to an emerging evidence base which supports a second-order hierarchical model of the structure of CPTSD (e.g. 9) when using validated measures and are in support of the ICD-11 proposals for CPTSD. Unlike Tay et al. (10), in the present study childhood trauma was found to be more strongly associated with DSO than with PTSD. Thus, CPTSD symptomatology compared with PTSD symptomatology in the current sample is consistent with the theoretical basis of CPTSD that exposure to childhood abuse increases the likelihood of a CPTSD, rather than a PTSD, response $(1,23)$.

Nevertheless, our study contained a number of limitations. Our sample consisted predominantly of people who had experienced childhood psychological trauma or had been multiply traumatized in childhood and adulthood. The present research and previous research (28) have indicated that childhood and multiple traumatization are risk factors for CPTSD (1). Therefore, our findings require replication with larger samples exposed to different types of traumatic events. A further limitation of this study is that we have not considered a number of possible alternative symptoms in our models that are highly prevalent in trauma populations, such as borderline symptoms and dissociation. Also, the measures used as indicators of DSO were not time-referenced, whereas the PCL-5 items used are based on how the participants felt in the last month. Finally, RSES has been developed as a measure of global self-esteem and not as a measure of negative self-concept following exposure to a traumatic event as per ICD-11 definition.

The relationship of dissociation to ICD-11 PTSD and CPTSD remains unknown. Dissociation has been described as an adaptive response to overwhelming peritraumatic negative affect such as fear or horror (e.g. 44). Events occurring in childhood tend to be more overwhelming because

Table 5. Pearson correlations between model 4 factor scores and trauma variables

\begin{tabular}{|c|c|c|c|c|c|c|}
\hline Model 4 Factor scores & CTO: Emotional abuse & CTO: Physical abuse & CTO: Sexual abuse & CTQ: Emotional neglect & CTQ: Physical neglect & Total LEC scores \\
\hline Re-experiencing & 0.300 & 0.272 & 0.308 & 0.245 & 0.254 & 0.244 \\
\hline Avoidance & 0.295 & 0.266 & 0.292 & 0.241 & 0.248 & 0.224 \\
\hline Threat & 0.245 & 0.219 & 0.231 & 0.195 & 0.200 & 0.241 \\
\hline Affective Dysregulation & 0.344 & 0.304 & 0.342 & 0.319 & 0.316 & 0.307 \\
\hline Negative Self-Concept & $-0.408^{*}$ & -0.358 & -0.403 & -0.378 & -0.360 & -0.295 \\
\hline Disturbances in Relationships & 0.306 & 0.266 & 0.305 & 0.287 & 0.266 & 0.274 \\
\hline 2nd Order - PTSD & 0.318 & 0.287 & 0.321 & 0.264 & 0.270 & 0.261 \\
\hline 2nd Order - DSO & 0.373 & 0.328 & 0.371 & 0.344 & 0.329 & 0.304 \\
\hline
\end{tabular}

All correlations significant $(P<0.05)$.

*This factor loading is negative as self-esteem reflects positive self-evaluation and the DSO factor is scored in a negative direction. 
children are less capable of organizing their responses to traumatic experiences coherently (45). Thus, dissociation may be more likely to fit a CPTSD profile rather than a PTSD profile, because CPTSD predominantly results from chronic, sustained and multiple types of traumatic exposures of interpersonal and childhood nature. DSM-5 (46) describes a dissociative subtype comprised of two symptoms (i.e. derealization and depersonalization), and it would be of interest to compare in future research the DSM-5 PTSD dissociative subtype to ICD-11 CPTSD in diverse trauma populations.

The relationship between BPD and CPTSD has been explored in a few studies (e.g. 28). It has been suggested that CPTSD can be distinguished from BPD by the nature of the constellation of symptoms and by differences in the risk for self-harm. For example, BPD does not require the presence of a traumatic event which is required in CPTSD. Furthermore, BPD is strongly characterized by fear of abandonment, shifting identity and frequent suicidal behaviours. In CPTSD, the fear of abandonment is not a requirement of the disorder and self-identity is consistently negative rather than shifting (47). More recent evidence suggests that four symptoms can clearly distinguish BPD from CPTSD: frantic efforts to avoid abandonment, unstable sense of self, unstable and intense interpersonal relationships and impulsiveness (28). However, further research is required on the association between BPD and CPTSD symptomatology.

Our findings have a number of implications for research, practice and policy. The ICD-11 proposals for PTSD and CPTSD create a need to further understand the biopsychosocial aetiological factors of complex traumatic stress reactions. There is emerging evidence, including the present study, that childhood traumatic life events are more closely related to complex traumatic stress reactions, but the pathways that lead to certain traumatic stress reactions, and the role of protective factors, remain unknown. This information is essential for the conceptualization and development of interventions to reverse traumatic impact. The findings that traumatic stress can be economically explained in terms of two higher order latent factors can simplify clinical practice with regard to assessing and treating individuals with traumatic stress. Nevertheless, it is important to emphasize that these findings support the proposals of ICD11 with regard to the structure of traumatic stress conditions, whereas further work is required to confirm the nature of the specific symptoms that comprise each factor. Our results suggest that screening for both PTSD and CPTSD in traumatized populations should be routine practice. There is adequate evidence for the treatment of PTSD but less so for CPTSD. The treatment of a unique CPTSD disorder may require alternative clinical interventions to the standard evidence-based methods of treating PTSD as per DSM-5 definitions (47). Multiphase therapeutic interventions have been developed for both adult (48) and child (49) CPTSD populations; however, further research is required on the effectiveness and acceptability of interventions for CPTSD.

\section{References}

1. Maercker A, Brewin CR, Bryant RA et al. Proposals for mental disorders specifically associated with stress in the ICD-11. Lancet 2013;381:1683-1685.

2. ReED GM. Toward ICD-11: improving the clinical utility of WHO's international classification of mental disorders. Prof Psychol Res 2010;41:457-464.

3. Forbes D, Lockwood E, Creamer M et al. Latent structure of the proposed ICD-11 post-traumatic stress disorder symptoms: implications for the diagnostic algorithm. Br J Psychiatry 2015;206:245-251.

4. Hansen M, Hyland P, Armour C, Shevlin M, Elklit A. Less is more? Assessing the validity of the ICD-11 model of PTSD across multiple trauma samples. Eur J Psychotraumatol 2015;6:28766.

5. TAY AK, ReEs S, Steel $Z$ et al. Six-year trajectories of post-traumatic stress and severe psychological distress symptoms and associations with timing of trauma exposure, ongoing adversity and sense of injustice: a latent transition analysis of a community cohort in conflictaffected Timor-Leste. BMJ Open 2016;6. doi: 10.1136/ bmjopen-2015-01020.

6. Glück TM, Knefel M, Tran US, Lueger-Schuster B. PTSD in ICD-10 and proposed ICD-11 in elderly with childhood trauma: prevalence, factor structure, and symptom profiles. Eur J Psychotraumatol 2016;7:29700.

7. Knefel M, Lueger-Schuster B. An evaluation of ICD-11 PTSD and complex PTSD criteria in a sample of adult survivors of childhood institutional abuse. Eur J Psychotraumatol 2013;4:22608.

8. Cloitre M, Garvert DW, Brewin CR et al. Evidence for proposed ICD-11 PTSD and complex PTSD: a latent profile analysis. Eur J Psychotraumatol 2013;4. 10.3402/ ejpt.v4i0.20706. http://doi.org/10.3402/ejpt.v4i0.20706

9. Hyland P, Shevlin M, Elklit A et al. An assessment of the construct validity of the ICD-11 proposal for complex posttraumatic stress disorder. Psychol Trauma 2017;9:1-9.

10. Tay AK, Rees S, Chen J, Kareth M, Silove D. The structure of post-traumatic stress disorder and complex posttraumatic stress disorder amongst West Papuan refugees. BMC Psychiatry 2015;15:111.

11. Derogatis LR. BSI brief symptom inventory. Administration, scoring, and procedures manual, 4th edn. Minneapolis, MN: National Computer Systems, 1993.

12. Briere J, Runtz M. The trauma symptom checklist (STC33): early data on a new scale. J Interpers Violence 1989;4:151-163.

13. Weathers FW, Litz BT, Keane TM et al. The PTSD Checklist for DSM-5 (PCL-5). Scale available from the 


\section{Shevlin et al.}

National Center for PTSD at http://www.ptsd.va.gov/pro fessional/assessment/adult-sr/ptsd-checklist.asp 2013.

14. Gratz KL, Roemer L. Multidimensional assessment of emotion regulation and dysregulation: development, factor structure, and initial validation of the difficulties in emotion regulation scale. J Psychopathol Behav Assess 2004;26:41-54.

15. Rosenberg M. Society and the adolescent self-image. Princeton, NJ: Princeton University Press, 1965.

16. Soldz S, Budman S, Demby A, Merry J. A short form of the inventory of interpersonal problems circumplex scales. Assessment 1995;2:53-63.

17. Conybeare D, Behar E, Solomon A et al. The PTSD Checklist-Civilian Version: reliability, validity, and factor structure in a nonclinical sample. J Clin Psychol 2012;68:699713.

18. Konecky B, Meyer EC, Kimbrel NA, Morissette SB. The structure of DSM-5 posttraumatic stress disorder symptoms in war veterans. Anxiety Stress Coping 2015;16:1-10.

19. Wilkins KC, Lang AJ, Norman SB. Synthesis of the psychometric properties of the PTSD checklist (PCL) military, civilian, and specific versions. Depress Anxiety 2011;28:596-606.

20. Neumann A, van Lier PA, Gratz KL, Koot HM. Multidimensional assessment of emotion regulation difficulties in adolescents using the Difficulties in Emotion Regulation Scale. Assessment 2010;17:138-149.

21. Schmitt DP, Allik J. Simultaneous administration of the Rosenberg Self-Esteem Scale in 53 nations: exploring the universal and culture-specific features of global selfesteem. J Pers Soc Psychol 2005;89:623-642.

22. Marsh HW, Scalas FL, Nagengast B. Longitudinal tests of competing factor structures for the Rosenberg Self-Esteem Scale: traits, ephemeral artifacts, and stable response styles. Psychol Assess 2010;22:366-381.

23. BARKHAM M. The IIP-32: a short version of the Inventory of Interpersonal Problems. Br J Clin Psychol 1996;35:21-35.

24. McEvoy PM, Burgess MM, Page AC, Nathan P, Fursland A. Interpersonal problems across anxiety, depression, and eating disorders: a transdiagnostic examination. Br J Clin Psychol 2013;52:129-147.

25. Bernstein DP, Fink L. Childhood trauma questionnaire: a retrospective self-report manual. San Antonio, TX: The Psychological Corporation, 1998.

26. Gray MJ, Litz BT, Hsu JL, Lombardo TW. Psychometric properties of the Life Events Checklist. Assessment 2004;11:330-341.

27. Matsunaga M. Item parcelling in structural equation modelling: a primer. Commun Methods Meas 2008;2:260-293.

28. Cloitre M, Garvert DW, Brewin CR, Bryant RA, MaerCKER A. Evidence for proposed ICD-11 PTSD and complex PTSD: a latent profile analysis. Eur J Psychotraumatol 2013;15:4.

29. Cloitre M, Garvert DW, Weiss B, Carlson EB. Distinguishing PTSD, Complex PTSD, and Borderline Personality Disorder: a latent class analysis. Eur J Psychotraumatol 2014;5. doi:10.3402/ejpt.v5.25097.
30. Ford JD, Courtors CA. Complex PTSD, affect dysregulation, and borderline personality disorder. Borderline Personal Disord Emot Dysregul 2014;1:9. doi:10.1186/20516673-1-9.

31. Muthén LK, Muthén BO. MPlus user's guide, 7th edn. Los Angeles, CA: Muthén \& Muthén, 1998-2013.

32. Yuan K, Bentler PM. Three likelihood-based methods for mean and covariance structure analysis with nonnormal missing data. Sociol Methodol 2000;30:167-202.

33. Rhemtulla M, Brosseau-Liard PE, Savalei V. When can categorical variables be treated as continuous? A comparison of robust continuous and categorical SEM estimation methods under suboptimal conditions. Psychol Methods 2012;17:354-373.

34. Beauducel A, Herzberg Py. On the performance of maximum likelihood versus means and variance adjusted weighted least squares estimation in CFA. Struct Equ Modeling 2006;13:186-203.

35. Bentler PM. Comparative fit indexes in structural models. Psychol Bull 1990;107:238-246.

36. TuCKer LR, Lewis C. The reliability coefficient for maximum likelihood factor analysis. Psychometrika 1973;38:110 .

37. Steiger JH. Structural model evaluation and modification: an interval estimation approach. Multivariate Behav Res 1990;25:173-180.

38. Jöreskog KG, Sörbom D. LISREL V: analysis of linear structural relationships by the method of maximum likelihood. Chicago: National Educational Resources, Inc., 1981.

39. Schwarz G. Estimating the dimension of a model. Ann Stat 1978;6:461-464.

40. RAFtery A. Bayesian model selection in social research. Sociol Methodol 1995;25:111-163.

41. MuthÉN BO. Mplus technical appendices; Appendix 11. Los Angeles, CA: Muthén \& Muthén. 1998-2004.

42. TANAKA JS. "How big is big enough?" Sample size and goodness of fit in structural equation models with latent variables. Child Dev 1987;58:134-146.

43. KLINE RB. Principles and practice of structural equation modeling. New York: Guilford Press, 2001.

44. BRIERE J. Child abuse trauma: theory and treatment of the lasting effects. Newbury Park, CA: Sage; 1992.

45. VAN DER KolK BA. Child abuse and victimization. Psychiatric Ann 2005;35:376-378.

46. American Psychiatric Association. Diagnostic and statistical manual of mental disorders, 5th edn. Arlington, VA: American Psychiatric Publishing, 2013.

47. Cloitre M, Courtois CA, Charuvastra A, Carapezza R. Treatment of complex PTSD: results of the ISTSS expert clinician survey on best practices. J Trauma Stress 2011;24:615-627.

48. Ford JD. Complex PTSD: research directions for nosology/assessment, treatment, and public health. Eur J Psychotraumatol 2015;6:27584.

49. Connor DF, Ford JD, Arnsten AF, Greene CA. An update on posttraumatic stress disorder in children and adolescents. Clin Pediatr 2015;54:517-528. 